\title{
How nanotechnology imaging can be used in kidney disease: an interview with $\mathrm{Dr}$ Mengxiao Yu
}

\author{
Mengxiao $\mathrm{Yu}^{*, 1,2}$ \\ ${ }^{1}$ Department of Chemistry and Biochemistry, The University of Texas at Dallas, 800 West Campbell Road, Richardson, TX 75080 , \\ USA \\ ${ }^{2}$ ClearNano, Inc., Waterview Parkway, Suite 1.202, Dallas, TX 75252, USA \\ *Author for correspondence: mengxiao.yu@utdallas.edu
}

\begin{abstract}
Author biography: Dr Yu currently is a research assistant professor at University of Texas at Dallas and is the Chief Scientist and Chief Executive Officer of ClearNano, Inc. She received her B.S. in Chemistry from Beijing Normal University in China in 2004, and her PhD in Chemistry from Fudan University in 2009. After that she joined Dr Jie Zheng's group at University of Texas at Dallas as a postdoctoral fellow and was promoted to research scientist in 2014 and research assistant professor in 2015. Her current research area comprises renal nanomedicine, biomedical imaging, luminescent gold nanoparticles and kidney disease detection.
\end{abstract}

First draft submitted: 21 September 2018; Accepted for publication: 26 October 2018; Published online: 4 December 2018

Keywords: functional imaging • kidney disease $\bullet$ nanomedicine

Could you please begin by introducing yourself, your academic background \& your work to date?

(1) I am a chemist, who is developing imaging agents for disease diagnosis, in particular for kidney disease.

(2) Current positions: I am a Research Assistant Professor at Department of Chemistry and Biochemistry at University of Texas at Dallas, and also Chief Scientist and CEO of ClearNano, Inc., which is dedicated to developing and commercializing advanced nanotechnologies for early detection of kidney disease.

(3) Academic background: I received my PhD degree in Chemistry from Laboratory of Advanced Materials at Fudan University in Shanghai, China, and had postdoctoral training in analytical chemistry and nanomedicines at University of Texas at Dallas, USA.

(4) Work to date is mainly focused on developing novel contrast agents for in vitro and in vivo functional imaging.

Are there any colleagues or role models in the field who you particularly look up to or are inspired by?

I was very fortunate to have three advisors who inspired me with their passion for science and never stop learning attitude. I still remember the first time I met Professor Chunhui Huang, who is an inorganic chemist and was co-advisor of my $\mathrm{PhD}$ research, in her office. She strongly encouraged young women to work on science and shared her own experience with me all the time. Professor Fuyou Li, who is an inorganic and analytical chemist, guided my $\mathrm{PhD}$ research on developing novel luminescent probes for bioimaging. I am always motivated by his courage of opening a new field with well-known rare earth materials. My role models also include Professor Jie Zheng who has a strong, fearless mind to follow his own passion for science and is always willing to help young researchers to grow up and identify their potentials. Professor Zheng is a physical chemist by training and one of the pioneers in developing highly fluorescent metal nanoparticles. He currently works on the understanding of physiology on the nanoscale. He was my postdoctoral mentor and is also my colleague now. We have been working together on fully exploring the clinical potentials of renal clearable nanomedicines for a decade.

Future Medicine 
Please describe the work you are currently conducting on 'noninvasive fluorescence kidney functional imaging technology' which you recently presented at the American Chemical Society National Meeting in Boston 2018?

Kidney disease is known as 'a silent killer', which causes more deaths than breast and prostate cancers combined. However, since current biomarkers such as blood urea nitrogen and serum creatinine are insensitive to kidney function changes, the demand to develop new approaches for the early detection of kidney disease and monitor its progression even in the preclinical research is high. Nearly 6 years ago, we started with an idea of exploring the feasibility of using near-infrared emitting renal clearable gold nanoparticles as probes to noninvasively stage kidney diseases based on in vivo fluorescence imaging. While this seems easy right now, nobody else had ever done this before because conventional organic fluorophores on the market were not working. We first unraveled what the problems of conventional organic dyes in kidney imaging were and then applied renal clearable gold nanoparticles to address those long-standing challenges in the noninvasive fluorescence kidney functional imaging. By the end, we were able to use these gold nanoparticles to enhance kidney contrast more than 50 times and prolong the imaging time window by nearly three orders over conventional organic dyes. Due to the high kidney imaging contrast, we were able to even quantify different stages of kidney dysfunction. This work was published in Angewandte Chemie (International edition in English) in 2015 and 2016, and highlighted by Chemical \& Engineering News in 2016. Looking back, it was a great journey for me to explore a brand new field that I have never imagined before. It gave me great satisfaction to learn so many things.

What are the implications of this work on the nanomedicine field?

This work brought many implications to the field of nanomedicines and early diagnosis of kidney diseases. First, it enabled in vivo fluorescence imaging technique to serve as a powerful tool for studying kidney disease and its progression at low cost but high sensitivity. Since in vivo fluorescence imaging system is quite common, researchers will be able to readily unravel mechanisms of kidney diseases in the preclinical setting. It could also help Big Pharma to evaluate drug-induced nephrotoxicity. Since gold nanoparticles can be detected with $\mathrm{x}$-ray, this work also enables $\mathrm{x}$-ray as a powerful tool to evaluate kidney dysfunction at improved contrast of the kidney.

What do you see as the biggest area of growth for imaging technology in nanomedicine?

It is hard to say which one will be the biggest area, since different nanomedicines have different strengths in the diagnosis of disease. However, I would say that those nanoprobes that can be relatively rapidly eliminated out of the body through renal excretion are gaining huge attention in the past decade, since they have much less health hazards compared with those nonrenal clearable imaging nanoparticles. Actually, one of them, Cornell Dots (also known as ' $\mathrm{C}$ ' dots') for cancer imaging, is being tested in the clinical trials.

What do you hope to achieve in your career? What are your long-term scientific goals?

One big dream in my career is to eventually translate my findings from the lab into the markets or clinics so that more people can benefit from our discoveries. My long-term scientific goals include further advancing fundamental understanding of how nanoparticles interact with the normal and diseased kidney and how they are transported in the kidneys; further applying these understandings to improve the diagnosis and treatment of kidney disease.

In your opinion, what should be done to get more women involved in science \& retain them? Based on my personal experience, I would say that giving them opportunities to do cutting edge research, interact with and learn from the experts from different fields and serve as principal investigators to lead research teams to tackle some long-standing challenges in the field. It is a tough journey for us but is also a fruitful journey for us to discover our own potentials.

\section{Disclaimer}

The opinions expressed in this interview are those of the interviewee and do not necessarily reflect the views of Future Medicine Ltd.

Financial \& competing interests disclosure

We gratefully acknowledge the financial support from the National Institutes of Health (NIH, grant numbers R01DK103363 [JZ], R01DK115986 [JZ] and R43DK116368 [MY]) and Welch Research Foun- 
dation (grant number AT-1974-20180324 [JZ]). M Yu has financial interest in ClearNano, Inc. that is dedicated to developing and commercializing technologies for early diagnosis of kidney disease. No writing assistance was utilized in the production of this manuscript.

\section{References}

1. Yu M, Liu J, Ning X, Zheng J. High-contrast noninvasive imaging of kidney clearance kinetics enabled by renal clearable nanofluorophores. Angew. Chem. Int. Ed. 54, 15434-15438 (2015).

2. Yu M, Zhou J, Du B, Ning X, Authement C, Gandee L et al. Noninvasive staging of kidney dysfunction enabled by renal clearable luminescent gold nanoparticles. Angew. Chem. Int. Ed. 55, 2787-2791 (2016).

3. Xu J, Yu M, Carter P, Hernandez E, Dang A, Kapur P et al. In vivo x-ray imaging of transport of renal clearable gold nanoparticles in the kidneys. Angew. Chem. Int. Ed. 56, 13356-13360 (2017).

4. Du B, Jiang X, Das A, Zhou Q, Yu M, Jin R et al. Glomerular barrier behaves as an atomically precise bandpass filter in a sub-nanometer regime. Nat. Nanotechnol. 12, 1096 (2017).

5. Du B, Yu M, Zheng J. Transport and interactions of nanoparticles in the kidneys. Nat. Rev. Mater. 3, 358-374 (2018).

6. Yu M, Xu J, Zheng J. Renal clearable luminescent gold nanoparticles: from bench to clinics. Angew. Chem. Int. Ed. doi:10.1002/anie.201807847 (2018) (Epub ahead of print).

7. Yu M, Zheng J, et al. Clearance pathways and tumor targeting of imaging nanoparticles. ACS Nano. 9, 6655-6674 (2015). 


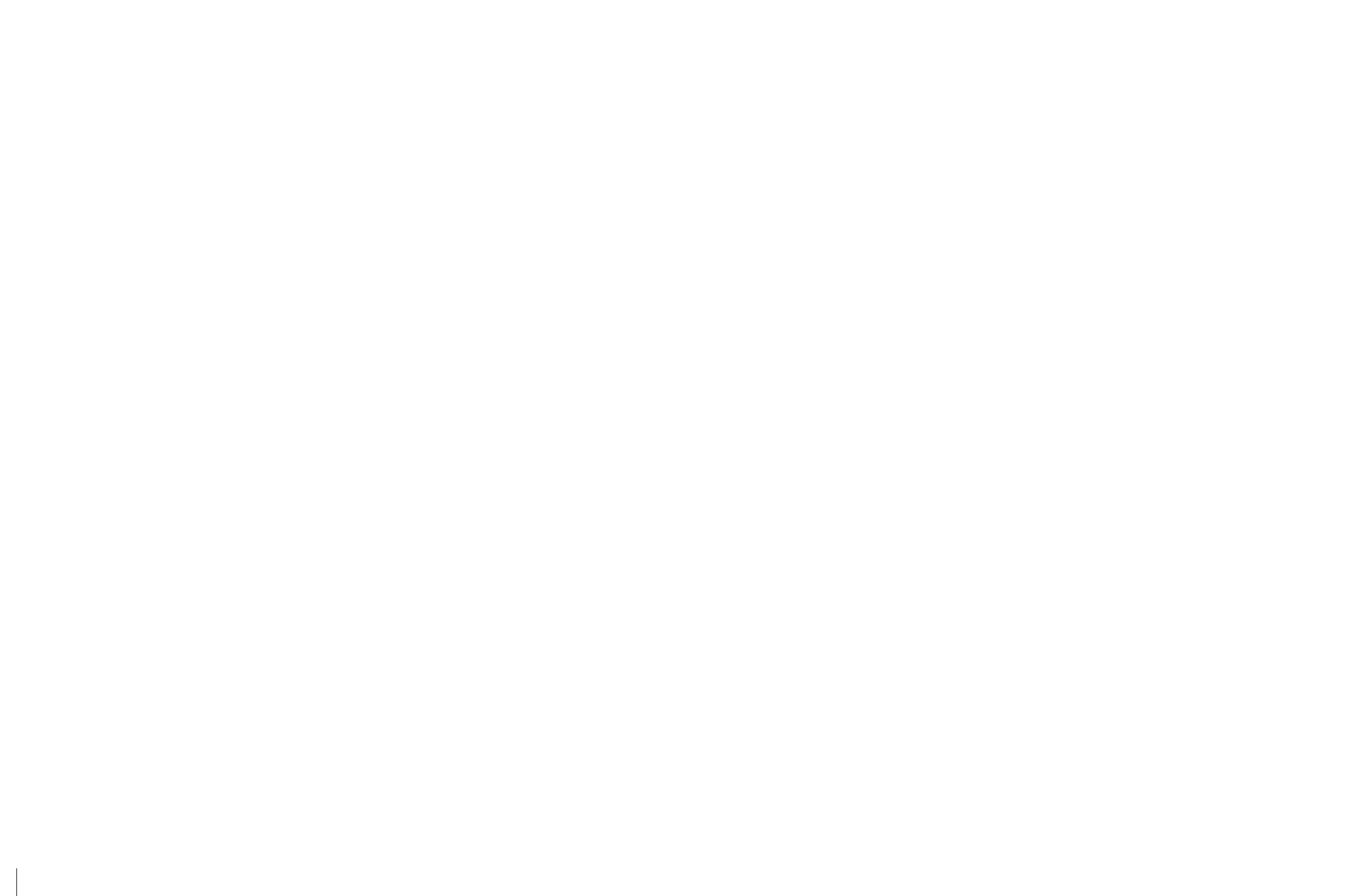

